\title{
Consonant random sets: structure and properties *
}

\author{
Enrique Miranda \\ Rey Juan Carlos University, Department of Informatics, Statistics and Telematics. \\ C-Tulipán, s/n 28933 Móstoles, Spain enrique.miranda@urjc.es
}

\begin{abstract}
In this paper, we investigate consonant random sets from the point of view of lattice theory. We introduce a new definition of consonancy and study its relationship with possibility measures as upper probabilities. This allows us to improve a number of results from the literature. Finally, we study the suitability of consonant random sets as models of the imprecise observation of random variables.
\end{abstract}

Keywords: Consonant random sets, lattice theory, possibility measures, upper probabilities, measurable selections.

\section{Introduction}

Random sets, or measurable multi-valued mappings, have gained a lot of attention in the past decades. They have been studied for instance within stochastic geometry ([16]), economy ([13]), or from the measure-theoretic point of view ([12]). Within random sets, those which are consonant constitute a subclass of particular interest, as the works in $[4,10,17,19]$ testify. In spite of all this work, there is not a unique definition of consonant random set; on the contrary, the term 'consonancy' has been used whenever there is some relationship of nestedness between the images of the multi-valued mapping. The different levels of this relationship, as well as other hypotheses that can be imposed on the random set, such as the initial and final spaces, or the topological characteristics of the images, have made of the term consonant random set a rather vague one.

In this paper, we try to get to the core of the notion of consonancy: we study this property not from the point of view of the order that we can consider in the images of the random set, but from the one we can induce on the elements of the initial space. We study the properties of this order within lattice theory, and use them to investigate a number of features of consonant random sets. Our main subject of interest is the relationship between consonant random sets and possibility measures. This relationship has been thoroughly studied in the literature, but the point of view we adopt in this paper allows us to easily characterise this property, generalising along the way some results from the literature. This is detailed in Sections 2 and 3. In Section 4 we investigate whether a consonant

\footnotetext{
* The research in this paper has been partially supported by MEC-DGI, grant numbers MTM2004-01269 and TSI2004-06801-C04-01. The scientific responsability rests with the author.
} 
random set can be used to model the imprecise observation of a random variable, and which would be the best tool in that case. Finally, Section 5 contains some conclusions and open problems on the matter at hand.

\section{Consonant random sets and lattice theory}

Let us introduce some basic concepts from random set theory.

Definition 1. Let $(\Omega, \mathcal{A}, P)$ be a probability space, $\left(X, \mathcal{A}^{\prime}\right)$ a measurable space and let $\Gamma: \Omega \rightarrow \mathcal{P}(X)$ be a multi-valued mapping. Given $A \in \mathcal{A}^{\prime}$, its upper inverse by $\Gamma$ is $\Gamma^{*}(A)=\{\omega \in \Omega: \Gamma(\omega) \cap A \neq \emptyset\}$. $\Gamma$ is said to be strongly measurable (or a random set) when $\Gamma^{*}(A)$ belongs to $\mathcal{A}$ for all $A \in \mathcal{A}^{\prime}$.

There are other conditions of measurability that can be considered on multivalued mappings (see a review in [14]). We have chosen the strong measurability ([22]), also called sometimes $\mathcal{B}$-measurability, because it allows us to define the upper probability of the random set, which will be one of the main points of interest in this paper.

Definition 2. [5] Let $(\Omega, \mathcal{A}, P)$ be a probability space, $\left(X, \mathcal{A}^{\prime}\right)$ a measurable space and consider a random set $\Gamma: \Omega \rightarrow \mathcal{P}(X)$. Given $A \in \mathcal{A}^{\prime}$, its upper probability is given by $P_{\Gamma}^{*}(A)=\frac{P\left(\Gamma^{*}(A)\right)}{P\left(\Gamma^{*}(X)\right)}$.

When there is no ambiguity about the random set we are working with, we shall denote $P^{*}=P_{\Gamma}^{*}$. The upper probability induced by a random set is $\infty$-alternating and lower continuous $([22])$.

Let us introduce next some notions of consonancy that can be considered on a random set. The idea underlying consonant random sets is the existence of some order in the set of images, normally by means of the inclusion operator. This order provides a common background to the images of the different elements of the initial space, so there is not contradiction between them (hence the term consonant). Although there are other conditions (see for instance $[17,19]$ ), the ones we recall here are the strongest and the most interesting ones for the purposes of this paper.

Definition 3. A random set $\Gamma: \Omega \rightarrow \mathcal{P}(X)$ is said to be

- antitone if $(\Omega, \mathcal{A}, P)=\left([0,1], \beta_{[0,1]}, \lambda_{[0,1]}\right)$, where $\beta_{[0,1]}$ and $\lambda_{[0,1]}$ denote respectively the Borel $\sigma$-field and the Lebesgue measure on $[0,1]$, and $x \leq$ $y \in[0,1] \Rightarrow \Gamma(x) \supseteq \Gamma(y)$.

- C1, if for any $\omega_{1}, \omega_{2} \in \Omega$, it is $\Gamma\left(\omega_{1}\right) \subseteq \Gamma\left(\omega_{2}\right)$ or $\Gamma\left(\omega_{2}\right) \subseteq \Gamma\left(\omega_{1}\right)$.

- C2, if the previous relation holds for any $\omega_{1}, \omega_{2}$ on $\Omega \backslash N$, where $N$ is a null subset of $\Omega$.

An isotone random set is one defined on $[0,1]$ such that the natural order is the same as we have in the images, that is, such that $x \leq y \Rightarrow \Gamma(x) \subseteq \Gamma(y)$. The dual notion of antitone random set allows us to relate consonant random sets 
to the $\alpha$-cuts of a fuzzy number [6] (but see also [21]). On the other hand, the more general condition $\mathrm{C} 1$ only requires the set of images to be totally ordered by the inclusion relationship, but it does not make any assumption on the initial space. The introduction of $\mathrm{C} 2$ random sets is due to the fact that the behaviour of a random set on a null subset of the initial space does not affect its upper probability (this will become clearer in Section 3). By Definition 3, it is clear that an antitone random set is in particular $\mathrm{C} 1$, and that a $\mathrm{C} 1$ random set is $\mathrm{C} 2$. Next, we are going to introduce an intermediate notion of consonancy that comes between $\mathrm{C} 1$ and antitone random sets. We shall denote this condition C0. It involves not only the existence of a total order on the initial space, but also some kind of 'continuity' in the way the images of the random set are nested.

Definition 4. A random set $\Gamma: \Omega \rightarrow \mathcal{P}(X)$ is said to be $\mathbf{C 0}$ if it satisfies the following two hypotheses:

- For any $\omega_{1}, \omega_{2} \in \Omega$, either $\Gamma\left(\omega_{1}\right) \subseteq \Gamma\left(\omega_{2}\right)$ or $\Gamma\left(\omega_{2}\right) \subseteq \Gamma\left(\omega_{1}\right)$.

- For any $A \subseteq \Omega$ there exists $B \subseteq A$ countable s.t. $\cap_{\omega \in A} \Gamma(\omega)=\cap_{\omega \in B} \Gamma(\omega)$.

The remainder of this paper is devoted to the study of the properties of $\mathrm{C} 0$ random sets, and the results we obtain will serve as a justification of their introduction. We shall see in particular that, even though a $\mathrm{C} 0$ random set is in particular $\mathrm{C} 1$, the converse is not true.

First, we are going to study the representation of consonant random sets in terms of lattices. We refer to $[2,9]$ for the definitions of the different concepts in lattice theory we shall use. Let $\Gamma$ be a $\mathrm{C} 1$ random set, and let us define, for any $\omega \in \Omega$, the class $[\omega]:=\left\{\omega^{\prime} \in \Omega \mid \Gamma\left(\omega^{\prime}\right)=\Gamma(\omega)\right\}$, and denote $\Omega^{*}:=\{[\omega] \mid \omega \in \Omega\}$. Let us define the relation $\preceq_{\Gamma}$ on $\Omega^{*}$ by

$$
\left[\omega_{1}\right] \preceq_{\Gamma}\left[\omega_{2}\right] \Leftrightarrow \Gamma\left(\omega_{1}\right) \subseteq \Gamma\left(\omega_{2}\right)
$$

Then, it is easy to see that $\left(\Omega^{*}, \preceq_{\Gamma}\right)$ is totally ordered (i.e., a chain). In fact, there is a one-to-one correspondence between chains and lattices induced by $\mathrm{C} 1$ random sets: given a chain $(\Omega, \preceq)$, it suffices to consider the multi-valued mapping $\Gamma: \Omega \rightarrow \mathcal{P}(\Omega)$ given by $\Gamma(\omega)=\left\{\omega^{\prime} \in \Omega: \omega^{\prime} \preceq \omega\right\}^{1}$. Then, the chain $\left(\Omega^{*}, \preceq_{\Gamma}\right)$ induced by $\Gamma$ coincides with $(\Omega, \preceq)$. We deduce from this correspondence that the lattice induced by a $\mathrm{C} 1$ random set is not complete in general. Nevertheless, when it is complete, it is a continuous lattice.

Definition 5. [9] Given a lattice $(\Omega, \preceq), \omega_{1}, \omega_{2} \in \Omega$, we say that $\omega_{1}$ is way below $\omega_{2}$ and denote $\omega_{1} \ll \omega_{2}$ when $\forall D \subseteq \Omega$ s.t. $\omega_{2} \preceq \sup D, \exists d \in D$ s.t. $\omega_{1} \preceq d$. $(\Omega, \preceq)$ is continuous if it is complete and $\omega=\sup \left\{\omega^{\prime} \ll \omega\right\}$ for any $\omega \in \Omega$.

Proposition 1. If $\left(\Omega^{*}, \preceq_{\Gamma}\right)$ is a complete lattice, then it is continuous.

\footnotetext{
${ }^{1}$ We can easily make $\Gamma$ a random set by considering $\mathcal{A}=\mathcal{A}^{\prime}=\mathcal{P}(\Omega)$ and $P$ a
} degenerate probability distribution on some $\omega_{0} \in \Omega$. 
Proof. Consider $\omega \in \Omega^{*}$. If $\omega \ll \omega$, the condition holds trivially. Otherwise, given $\omega^{\prime} \prec_{\Gamma} \omega$, it follows from the definition that $\omega^{\prime} \ll \omega$. We deduce that $\left\{\omega^{\prime} \prec_{\Gamma} \omega\right\}=\left\{\omega^{\prime} \ll \omega\right\}$, and then it is clear that $\omega=\sup \left\{\omega^{\prime} \ll \omega\right\}$.

The random set $\Gamma$ induces a multi-valued mapping $\Gamma^{\prime}: \Omega^{*} \rightarrow \mathcal{P}(X)$ by $\Gamma^{\prime}([\omega])=\Gamma(\omega)$. If we consider on $\mathcal{P}(X)$ the partial order given by the inclusion, then $\Gamma^{\prime}$ is an homomorphism, and it is an isomorphism if we restrict the final space to $\operatorname{Im}(\Gamma)=\operatorname{Im}\left(\Gamma^{\prime}\right)$. Moreover, the class of the upper inverses of the elements of $\mathcal{A}^{\prime}$ can be characterised in terms of filters of $\Omega^{*}$. Let us consider $\mathcal{H}=\left\{[\omega] \in \Omega^{*}: \Gamma(\omega)=\cup_{\left[\omega^{\prime}\right] \prec_{\Gamma}[\omega]} \Gamma\left(\omega^{\prime}\right)\right\}$ and let us define the sets of filters

$$
\mathcal{H}_{1}:=\left\{\left\{\left[\omega^{\prime}\right] \succeq_{\Gamma}[\omega]\right\}:[\omega] \in \mathcal{H}\right\}, \mathcal{H}_{2}:=\left\{B \subseteq \Omega^{*}: B \text { is a filter }, B \notin \mathcal{H}_{1}\right\}
$$

Proposition 2. For any $A \in \mathcal{A}^{\prime}, \Gamma^{\prime *}(A) \in \mathcal{H}_{2}$. If moreover $\mathcal{A}^{\prime}=\mathcal{P}(X)$, then $\left\{\Gamma^{\prime *}(A): A \subseteq X\right\}=\mathcal{H}_{2}$.

Proof. Consider $A \in \mathcal{A}^{\prime}$. Given $[\omega] \in \Gamma^{\prime *}(A),\left[\omega^{\prime}\right] \succ_{\Gamma}[\omega]$, it is $\emptyset \neq \Gamma(\omega) \cap A \subseteq$ $\Gamma\left(\omega^{\prime}\right) \cap A$, whence $\left[\omega^{\prime}\right] \in \Gamma^{\prime *}(A)$. Since $\left(\Omega^{*}, \preceq_{\Gamma}\right)$ is totally ordered, we deduce that $\Gamma^{\prime *}(A)$ is a filter. Assume ex-absurdo that $\Gamma^{\prime *}(A)=\left\{[\omega] \succeq_{\Gamma}\left[\omega_{0}\right]\right\}$ for some $\left[\omega_{0}\right] \in \mathcal{H}$. Then, there exists $x \in \Gamma\left(\omega_{0}\right) \cap A=\left(\cup_{[\omega] \prec_{\Gamma}\left[\omega_{0}\right]} \Gamma(\omega)\right) \cap A$, whence there exists $\left[\omega_{1}\right] \prec_{\Gamma}\left[\omega_{0}\right]$ s.t. $x \in \Gamma\left(\omega_{1}\right)$. This means that $\left\{[\omega] \succeq_{\Gamma}\left[\omega_{1}\right]\right\} \subseteq \Gamma^{\prime *}(\{x\}) \subseteq$ $\Gamma^{\prime *}(A)=\left\{[\omega] \succeq_{\Gamma}\left[\omega_{0}\right]\right\} \subsetneq\left\{[\omega] \succeq_{\Gamma}\left[\omega_{1}\right]\right\}$, a contradiction.

Assume now $\mathcal{A}^{\prime}=\mathcal{P}(X)$, and consider $B \in \mathcal{H}_{2}$. Let $A=\left(\cup_{[\omega] \notin B} \Gamma(\omega)\right)^{c}$. Given $[\omega] \notin B, \Gamma^{\prime}([\omega]) \cap A=\Gamma(\omega) \cap\left(\cup_{\left[\omega^{\prime}\right] \notin B} \Gamma\left(\omega^{\prime}\right)\right)^{c}=\emptyset$, whence $\Gamma^{\prime *}(A) \subseteq B$. Now, if there exists $\left[\omega_{0}\right] \in B \backslash \Gamma^{\prime *}(A)$,

$$
\begin{aligned}
A \subseteq \Gamma\left(\omega_{0}\right)^{c} \subseteq \cap_{[\omega] \notin B} \Gamma(\omega)^{c}=A \Rightarrow & \Gamma\left(\omega_{0}\right)=\cup_{[\omega] \notin B} \Gamma(\omega) \\
& \Rightarrow \Gamma\left(\omega_{0}\right)=\cup_{[\omega] \prec_{\Gamma}\left[\omega_{0}\right]} \Gamma(\omega) \Rightarrow\left[\omega_{0}\right] \in \mathcal{H} .
\end{aligned}
$$

Hence, $B=\left\{[\omega] \succeq_{\Gamma}\left[\omega_{0}\right]\right\} \in \mathcal{H}_{1}$, a contradiction. Consequently, $\Gamma^{\prime *}(A)=B$, and we deduce the desired equality.

This proposition will be useful in the next section.

\section{Consonant random sets and possibility measures}

One of the main features of consonant random sets is their connection with supremum-preserving set functions. These set functions have appeared in the literature under a number of different names (see [23, 26, 27]), although since Zadeh's paper [28] they have been mostly referred to with the term possibility measure. This designation is due to their usefulness for modelling linguistic uncertainty $([7,28])$.

Definition 6. Let $(X, \mathcal{A})$ be a measurable space. A monotone and normalised set function $\Pi: \mathcal{A} \rightarrow[0,1]$ is called maxitive if for any $A_{1}, \ldots, A_{n} \in \mathcal{A}$, $\Pi\left(\cup_{i=1}^{n} A_{i}\right)=\max _{i=1, \ldots, n} \Pi\left(A_{i}\right)$, and it is said to be a possibility measure when for any family $\left(A_{i}\right)_{i \in I}$ of elements of $\mathcal{A}$ such that $\cup_{i \in I} A_{i} \in \mathcal{A}$, it is $\Pi\left(\cup_{i \in I} A_{i}\right)=\sup _{i \in I} \Pi\left(A_{i}\right)$. 
When all the singletons belong to the $\sigma$-field $\mathcal{A}$, a possibility measure $\Pi$ is characterised by its possibility distribution $\pi: X \rightarrow[0,1]$, which is given by $\pi(x)=\Pi(\{x\})$. Then, it is $\Pi(A)=\sup _{x \in A} \pi(x)$ for any $A \in \mathcal{A}$.

The relationship between possibility measures and consonant random sets is rather intuitive if we restrict ourselves to finite spaces: a monotone and normalized set function on a finite space is a possibility measure if and only if its focal elements are nested ([24]); on the other hand, the focal elements of the upper probability of a random set are those subsets of the final space whose inverses have probability non-zero. Taking this into account, it is easy to prove the following:

Proposition 3. [8, 17] Let $(\Omega, \mathcal{A}, P)$ be a probability space, $(X, \mathcal{P}(X))$ a finite space and let $\Gamma: \Omega \rightarrow \mathcal{P}(X)$ be a random set. Then, $P^{*}$ is a possibility measure if and only if $\Gamma$ is $C 2$.

There are many works in the literature devoted to the investigation of this equality in the infinite case; that is, whether the upper probability of a consonant random set is always a possibility measure and whether when the upper probability is a possibility measure the random set is necessarily consonant. In [17], we showed that a $\mathrm{C} 1$ random set does not induce in general a possibility measure, and that a random set inducing a possibility measure need not be C2. Nevertheless, there exist a number of sufficient conditions for these implications. We summarise the most important ones in the following theorem:

Theorem 1. 1. ([4]) If $\Gamma$ is antitone, then $P^{*}$ is a possibility measure.

2. ([19]) If $\Gamma$ is $C 2, P^{*}$ is maxitive.

3. ([19]) If $\Gamma$ is compact on a Polish space, or closed on a $\sigma$-compact metric space, then $P^{*}$ is a possibility measure $\Longleftrightarrow \Gamma$ is $C 2$.

In this paper, we are going to study this problem for the new condition of consonancy we have introduced, and we are going to take advantage of the representation of consonant random sets in terms of lattices made in the previous section. Let us define $\mathcal{A}_{1}^{\prime}=\left\{A \in \mathcal{A}^{\prime}: \Gamma^{\prime *}(A) \neq\left\{\left[\omega^{\prime}\right] \succeq_{\Gamma}[\omega]\right\} \forall[\omega] \in \Omega^{*}\right\}$. The following fairly straightforward result reduces the problem of studying if the upper probability is a possibility measure to arbitrary unions in $\mathcal{A}_{1}^{\prime}$.

Proposition 4. $P^{*}$ is a possibility measure if and only if for any $\left(A_{i}\right)_{i \in I}$ s.t. $\cup_{i \in I} A_{i} \in \mathcal{A}_{1}^{\prime}, P^{*}\left(\cup_{i \in I} A_{i}\right)=\sup _{i \in I} P^{*}\left(A_{i}\right)$.

Proof. Consider $\left(A_{i}\right)_{i \in I}$ in $\mathcal{A}^{\prime}$ s.t. $A:=\cup_{i \in I} A_{i}$ belongs to $\mathcal{A}^{\prime} \backslash \mathcal{A}_{1}^{\prime}$. Then, there exists $\left[\omega_{0}\right]$ s.t. $\Gamma^{*}(A)=\left\{[\omega] \succeq_{\Gamma}\left[\omega_{0}\right]\right\}$. Now, $\Gamma\left(\omega_{0}\right) \cap A \neq \emptyset \Rightarrow \exists i_{0} \in I$ s.t. $\Gamma\left(\omega_{0}\right) \cap A_{i_{0}} \neq \emptyset$, whence $\Gamma^{*}(A)=\Gamma^{\prime *}\left(A_{i_{0}}\right)$ and $P^{*}(A)=\sup _{i \in I} P^{*}\left(A_{i}\right)$. We conclude that $P^{*}$ is a possibility measure if and only if it is supremum-preserving for arbitrary collections of elements of $\mathcal{A}^{\prime}$ whose union belongs to $\mathcal{A}_{1}^{\prime}$.

Taking this proposition into account, we can establish the following theorem: 
Theorem 2. Let $(\Omega, \mathcal{A}, P)$ be a probability space, $(X, \mathcal{P}(X))$ a measurable space and $\Gamma: \Omega \rightarrow \mathcal{P}(X)$ a C1 random set s.t. $\left(\Omega^{*}, \preceq_{\Gamma}\right)$ is a complete chain. Then, $P^{*}$ is a possibility measure if and only if $\forall A \in \mathcal{A}_{1}^{\prime}$ s.t. $\cap_{[\omega] \in \Gamma^{\prime *}(A)} \Gamma(\omega) \cap A=\emptyset$, there exists some countable $\left\{\left[\omega_{n}\right]\right\}_{n} \subseteq \Gamma^{\prime *}(A)$ s.t. $P^{*}(A)=P\left(\cup_{n}\left\{[\omega] \succeq_{\Gamma}\left[\omega_{n}\right]\right\}\right)$.

Proof. Since $\left(\Omega^{*}, \preceq_{\Gamma}\right)$ is complete, given $A \in \mathcal{A}_{1}^{\prime}$, there exists some $\left[\omega_{A}\right] \in \Omega^{*}$ s.t. $\Gamma^{*}(A)=\left\{[\omega] \succ_{\Gamma}\left[\omega_{A}\right]\right\}$. Now, if there is some $x \in \cap_{[\omega] \succ_{\Gamma}\left[\omega_{A}\right]} \Gamma(\omega) \cap A$, then trivially $\Gamma^{\prime *}(\{x\})=\Gamma^{*}(A)$ and $P^{*}(A)=P^{*}(\{x\})$. Hence, $P^{*}$ will be a possibility measure if and only if $P^{*}(A)=\sup _{x \in A} P^{*}(\{x\})$ when $A \in \mathcal{A}_{1}^{\prime}$ and $\cap_{[\omega] \succ_{\Gamma}\left[\omega_{A}\right]} \Gamma(\omega) \cap A=\emptyset$.

$(\Rightarrow)$ If there exists $x \in A$ s.t. $P^{*}(\{x\})=\sup _{y \in A} P^{*}(\{y\})=P^{*}(A)$ then, since $\left(\Omega^{*}, \preceq_{\Gamma}\right)$ is complete and $\Gamma^{\prime *}(\{x\})$ is a filter, there exists $\left[\omega_{x}\right] \succeq_{\Gamma}\left[\omega_{A}\right]$ s.t. $\left\{\left[\omega^{\prime}\right] \succ_{\Gamma}\left[\omega_{x}\right]\right\} \subseteq \Gamma^{\prime *}(\{x\}) \subseteq\left\{\left[\omega^{\prime}\right] \succeq_{\Gamma}\left[\omega_{x}\right]\right\}$. There are three possibilities: if $\left[\omega_{x}\right] \in \Gamma^{\prime *}(\{x\})$, the result holds. If $\left[\omega_{x}\right] \notin \Gamma^{*}(\{x\})$ but $\left[\omega_{x}\right] \succ_{\Gamma}\left[\omega_{A}\right]$, then we have $P^{*}(A)=P\left(\left\{\left[\omega^{\prime}\right] \succ_{\Gamma}\left[\omega_{A}\right]\right\}\right)=P\left(\left\{\left[\omega^{\prime}\right] \succeq_{\Gamma}\left[\omega_{x}\right]\right\}\right)$, and the result holds. And finally, if $\left[\omega_{x}\right]=\left[\omega_{A}\right]$, then $x \in \Gamma(\omega)$ for any $[\omega] \succ_{\Gamma}\left[\omega_{A}\right]$, a contradiction with $\cap_{[\omega] \succ_{\Gamma}\left[\omega_{A}\right]} \Gamma(\omega)=\emptyset$.

Assume now that $P^{*}(\{x\})<P^{*}(A) \forall x \in A$; then there exists a sequence $\left\{x_{n}\right\}_{n}$ s.t. $P^{*}\left(\left\{x_{n}\right\}\right)_{n}$ converges to $\sup _{y \in A} P^{*}(\{y\})=P^{*}(A)$, and such that $P^{*}\left(\left\{x_{n}\right\}\right)<P^{*}\left(\left\{x_{n+1}\right\}\right) \forall n$. For any $n$, there exists some $\left[\omega_{n}\right] \succ_{\Gamma}\left[\omega_{A}\right]$ s.t. $\left\{\left[\omega^{\prime}\right] \succ_{\Gamma}\left[\omega_{n}\right]\right\} \subseteq \Gamma^{*}\left(\left\{x_{n}\right\}\right) \subseteq\left\{\left[\omega^{\prime}\right] \succeq_{\Gamma}\left[\omega_{n}\right]\right\}$, and then $P^{*}(A)=P\left(\left\{[\omega] \succ_{\Gamma}\right.\right.$ $\left.\left[\omega_{A}\right]\right)=P^{*}\left(\left\{x_{n}\right\}_{n}\right)=P\left(\cup_{n}\left\{[\omega] \succeq_{\Gamma}\left[\omega_{n}\right]\right\}\right.$

$(\Leftarrow)$ Consider $A \in \mathcal{A}_{1}^{\prime}$, and let $\left\{\left[\omega_{n}\right]\right\}_{n} \subseteq \Gamma^{\prime *}(A)$ s.t. $P^{*}(A)=P\left(\cup_{n}\left\{[\omega] \succeq_{\Gamma}\right.\right.$ $\left.\left.\left[\omega_{n}\right]\right\}\right)$. For any natural number $n$, take $x_{n} \in \Gamma\left(\omega_{n}\right) \cap A$. Then, $P^{*}\left(\left\{x_{n}\right\}_{n}\right) \geq$ $P\left(\cup_{n}\left\{[\omega] \succeq_{\Gamma}\left[\omega_{n}\right]\right\}\right)=P^{*}(A)$, and since Theorem 1 implies that $P^{*}$ is maxitive, $P^{*}(A)=\sup _{n} P^{*}\left(\left\{x_{n}\right\}\right)$.

Next, we use the ideas in this theorem to establish the main result of this paper. It establishes some relationships between different conditions of consonancy and possibility measures as upper probabilities, under more general conditions than the ones in Theorem 2: note that we do not require here neither the chain on the initial space to be complete nor the final $\sigma$-field to agree with $\mathcal{P}(X)$.

Theorem 3. Let $\Gamma$ be a C1 random set. Then, each of the following hypotheses implies the next:

1. $\Gamma$ is antitone.

2. $\Gamma$ is $C 0$.

3. For any $A \in \mathcal{A}_{1}^{\prime}$ there exists some $\left\{\left[\omega_{n}\right]\right\}_{n} \subseteq \Gamma^{\prime *}(A)$ such that $\Gamma^{\prime *}(A)=$ $\cup_{n}\left\{[\omega] \succeq_{\Gamma}\left[\omega_{n}\right]\right\}$.

4. $P^{*}$ is a possibility measure.

Proof. We start showing that $(1 \Rightarrow 2)$. Let $\Gamma$ be antitone, and consider $A \subseteq[0,1]$. If $\inf A$ belongs to $A$, then $\cap_{\omega \in A} \Gamma(\omega)=\Gamma(\inf A)$. If $\inf A \notin A$, let $\left\{\omega_{n}\right\}_{n}$ be a sequence of elements of $A$ that converges to inf $A$. Then, $\cap_{\omega \in A} \Gamma(\omega)=\cap_{n} \Gamma\left(\omega_{n}\right)$. Since any antitone random set is $\mathrm{C} 1$, we conclude that $\Gamma$ is $\mathrm{C} 0$. 
Let us show next that $(2 \Rightarrow 3)$. Consider $A \in \mathcal{A}_{1}^{\prime}$. Then, there exists $\left\{\left[\omega_{n}\right]\right\}_{n} \subseteq \Gamma^{\prime *}(A)$ s.t. $\cap_{[\omega] \in \Gamma^{\prime *}(A)} \Gamma(\omega)=\cap_{n} \Gamma\left(\omega_{n}\right)$. It is clear that $\cup_{n}\left\{[\omega] \succeq_{\Gamma}\right.$ $\left.\left[\omega_{n}\right]\right\} \subseteq \Gamma^{\prime *}(A)$, because this is a filter from Proposition 2. Assume ex-absurdo that there exists $\left[\omega_{0}\right] \in \Gamma^{\prime *}(A)$ s.t. $\left[\omega_{0}\right] \prec_{\Gamma}\left[\omega_{n}\right] \forall n$. Then, $\cap_{[\omega] \in \Gamma^{\prime *}(A)} \Gamma(\omega) \subseteq$ $\Gamma\left(\omega_{0}\right) \subseteq \cap_{n} \Gamma\left(\omega_{n}\right)=\cap_{[\omega] \in \Gamma^{\prime *}(A)} \Gamma(\omega)$, whence $\cap_{[\omega] \in \Gamma^{\prime *}(A)} \Gamma(\omega)=\Gamma\left(\omega_{0}\right)$. This means that $\Gamma^{\prime *}(A)=\left\{\left[\omega^{\prime}\right] \succeq_{\Gamma}\left[\omega_{0}\right]\right\}$, which contradicts $A \in \mathcal{A}_{1}^{\prime}$. Hence, $\Gamma^{\prime *}(A)=$ $\cup_{n}\left\{\left[\omega^{\prime}\right] \succeq_{\Gamma}\left[\omega_{n}\right]\right\}$.

Finally, we prove $(3 \Rightarrow 4)$. Consider $\left(A_{i}\right)_{i \in I} \in \mathcal{A}^{\prime}$ s.t. $\cup_{i \in I} A_{i}=A \in \mathcal{A}_{1}^{\prime}$. Then, condition (3) implies the existence of $\left\{\left[\omega_{n}\right]\right\}_{n} \subseteq \Gamma^{\prime *}(A)$ s.t. $\Gamma^{\prime *}(A)=$ $\cup_{n}\left\{[\omega] \succeq_{\Gamma}\left[\omega_{n}\right]\right\}$. For every $n$, there exists some $A_{n} \in\left(A_{i}\right)_{i \in I}$ s.t. $\Gamma\left(\omega_{n}\right) \cap A_{n} \neq \emptyset$, whence $\Gamma^{\prime *}(A)=\cup_{n}\left\{[\omega] \succeq_{\Gamma}\left[\omega_{n}\right]\right\}=\cup_{n} \Gamma^{\prime *}\left(A_{n}\right)$. As a consequence, we also have $\Gamma^{*}(A)=\cup_{n} \Gamma^{*}\left(A_{n}\right)$. Since $P^{*}$ is maxitive from Theorem 1 and lower continuous, we conclude that $P^{*}(A)=\sup _{n} P^{*}\left(A_{n}\right)=\sup _{i \in I} P^{*}\left(A_{i}\right)$. Applying Proposition $4, P^{*}$ is a possibility measure.

Example 1. It is easy to construct examples showing that the converses of these implications are not true in general: for the first, consider $\Gamma:[1,2] \rightarrow \mathcal{P}([1,2])$ given by $\Gamma(\omega)=[\omega, 2]$; the second and third counterexamples can be obtained by suitably modifying the $\mathrm{C} 1$ random set $\Gamma$ in $\left[17\right.$, Example 5] s.t. $P^{*}$ is not a possibility measure: for the first, consider $\mathcal{A}^{\prime}=\{\emptyset, \mathcal{P}(X)\}$, and $\Gamma_{1}:[0,1] \rightarrow$ $\mathcal{P}([0,1])$ given by $\Gamma_{1}(\omega)=\Gamma(\omega) \cup\{0\} \forall \omega \neq 0, \Gamma_{1}(0)=\{0\}$; for the second, take $\Gamma_{2}=\Gamma$ but with a degenerate probability measure on the initial space.

We deduce that an antitone random set is not necessarily $\mathrm{C} 0$, and consequently Theorem 3 generalises the first point of Theorem 1. On the other hand, since a $\mathrm{C} 1$ random set does not induce a possibility measure ([17]), we deduce that this condition of consonancy is weaker than that of $\mathrm{C} 0$.

As we said before, a random set inducing a possibility measure is not necessarily C2 [17], and, consequently, it is not C0 either. Nevertheless, it may be useful to study the representability of a possibility measure in terms of a consonant random set. Goodman proved in [10] that for any possibility measure $\Pi$ on a measurable space $(X, \mathcal{P}(X))$ there exists an antitone random set whose upper probability is $\Pi$. In [19], we considered the problem of the representability when we fix also the initial space. We proved that for any random set $\Gamma$ inducing a possibility measure there is a $\mathrm{C} 1$ random set $\Gamma_{1}$ defined between the same spaces and with the same upper probability. We show next that we may even require $\Gamma_{1}$ to be $\mathrm{C} 0$ :

Proposition 5. Let $(\Omega, \mathcal{A}, P)$ be a probability space, $(X, \mathcal{P}(X))$ a measurable space and $\Gamma: \Omega \rightarrow \mathcal{P}(X)$ a random set such that $P_{\Gamma}^{*}$ is a possibility measure. Then, there exists a $C 0$ random set $\Gamma_{1}: \Omega \rightarrow \mathcal{P}(X)$ such that $P_{\Gamma_{1}}^{*}=P_{\Gamma}^{*}$.

Proof. Let us define $C_{x}:=\left\{y \mid P^{*}(\{y\}) \geq P^{*}(\{x\})\right\}$ for any $x \in X$, and $\Gamma_{1}: \Omega \rightarrow$ $\mathcal{P}(X)$ by $\Gamma_{1}(\omega)=\cup_{x \in \Gamma(\omega)} C_{x}$. We check in [19, Theorem 4.7] that $\Gamma_{1}$ is strongly measurable, $\mathrm{C} 1$ and that $P_{\Gamma_{1}}^{*}=P_{\Gamma}^{*}$. It remains then to verify that it is also C0. Let us consider $A \subseteq \Omega$, and let us denote $z_{A}=\sup _{\omega \in A} \inf _{x \in \Gamma(\omega)} P^{*}(\{x\})$. 
From the definition of $\Gamma_{1}$ we deduce that there are only two alternatives: either $\cap_{\omega \in A} \Gamma_{1}(\omega)=\left\{y: P^{*}(\{y\}) \geq z_{A}\right\}$, and then given a sequence $\left\{\omega_{n}\right\}_{n}$ of elements of $A$ such that $z_{n}=\inf _{x \in \Gamma\left(\omega_{n}\right)} P^{*}(\{x\})$ converges to $z_{A}$, it is $\cap_{n} \Gamma_{1}\left(\omega_{n}\right) \subseteq$ $\cap_{n}\left\{y \mid P^{*}(\{y\}) \geq z_{n}\right\}=\left\{y \mid P^{*}(\{y\}) \geq z_{A}\right\}=\cap_{\omega \in A} \Gamma_{1}(\omega) ;$ or $\cap_{\omega \in A} \Gamma_{1}(\omega)=\{y:$ $\left.P^{*}(\{y\})>z_{A}\right\} \subsetneq\left\{y: P^{*}(\{y\}) \geq z_{A}\right\}$. In that case, given $y_{0}$ s.t. $P^{*}\left(\left\{y_{0}\right\}\right)=z_{A}$, there exists $\omega_{0} \in A$ s.t. $y_{0} \notin \Gamma_{1}\left(\omega_{0}\right)$, whence $\Gamma_{1}\left(\omega_{0}\right)=\left\{y \mid P^{*}(\{y\})>z_{A}\right\}=$ $\cap_{\omega \in A} \Gamma_{1}(\omega)$. We conclude in both cases that $\Gamma_{1}$ is $\mathrm{C} 0$.

To conclude this section, we represent in Figure 1 the relationships between the different conditions of consonancy we have considered and possibility and maxitive measures as upper probabilities. It follows from Example 1 and the examples in $[17,19]$ that none of the converses of these implications holds in general.



Fig. 1. Relationships between consonancy, $P^{*}$ possibility and $P^{*}$ maxitive.

\section{Consonant random sets as imprecise random variables}

Among the different interpretations given to random sets, one of the most important in the framework of uncertainty modelling is that of imprecise observations of random variables. This goes back to Kruse and Meyer [15]: we assume the existence of a measurable mapping $U_{0}: \Omega \rightarrow X$ which is observed with some imprecision, so that for any $\omega$ in the initial space all we know about $U_{0}(\omega)$ is that it belongs to some subset $\Gamma(\omega)$ of the final space ${ }^{2}$. We obtain then a multivalued mapping $\Gamma: \Omega \rightarrow \mathcal{P}(X)$, which, in case it satisfies the condition of strong measurability, is a random set.

Under such interpretation, our interest lies in the information we can recover about the 'original' random variable, $U_{0}$. All we know is that it belongs to the

\footnotetext{
${ }^{2}$ Hence, we will assume in this section that $\Gamma(\omega)$ is non-empty for all $\omega \in \Omega$.
} 
class

$$
S(\Gamma):=\{U: \Omega \rightarrow X \text { measurable, } U(\omega) \in \Gamma(\omega) \forall \omega\}
$$

of measurable selections of $\Gamma$, and consequently its distribution belongs to

$$
P(\Gamma):=\left\{P_{U}: U \in S(\Gamma)\right\} .
$$

In this section, we are going to study whether this interpretation is compatible with the one we have given to consonant random sets. For this, we must determine first if a consonant random set possesses measurable selections. This is what we prove in the following theorem:

Proposition 6. Let $(\Omega, \mathcal{A}, P)$ be a probability space, $\left(X, \mathcal{A}^{\prime}\right)$ a measurable space s.t. $\mathcal{A}^{\prime}$ contains the singletons and let $\Gamma: \Omega \rightarrow \mathcal{P}(X)$ be a $C 0$ random set. Then, $S(\Gamma) \neq \emptyset$.

Proof. If there is some $x \in \cap_{\omega \in \Omega} \Gamma(\omega)$, then the constant mapping on $x$ is trivially a measurable selection of $\Gamma$. Assume then that $\cap_{\omega \in \Omega} \Gamma(\omega)=\emptyset$. Since $\Gamma$ is C0, there exists a countable set $\left\{\omega_{n}\right\}_{n}$ such that $\cap_{n} \Gamma\left(\omega_{n}\right)=\cap_{\omega \in \Omega} \Gamma(\omega)$, and we may assume without loss of generality that $\Gamma\left(\omega_{n}\right) \subsetneq \Gamma\left(\omega_{n-1}\right)$ for all $n \geq 2$. Let us consider $x_{n} \in \Gamma\left(\omega_{n}\right) \backslash \Gamma\left(\omega_{n+1}\right)$ for every $n \geq 1$, and define

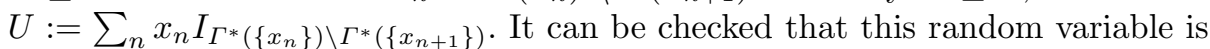
well defined (that is, $U(\omega) \neq \emptyset \forall \omega)$, and this implies that $U$ is a measurable selection of $\Gamma$.

As far as we know, a similar result for C1 random sets hasn't been established. On the other hand, it is easy to check that a random set inducing a possibility measure has an almost sure measurable selection (i.e., there exists $U: \Omega \rightarrow$ $X$ measurable such that $U(\omega) \in \Gamma(\omega)$ for all but a null subset of $\Omega$ ). These selections are sometimes used instead of everywhere selections (see for instance [12]); however, when we interpret a random set as a model of the imprecise observation of a random variable, we need to consider measurable mappings which are selections on all the elements of the initial space, and not just on a subset of probability one.

As we said before, if a random set $\Gamma$ models the imprecise observation of $U_{0}$, our information about $P_{U_{0}}$ is given by the class $P(\Gamma)$; although this is the most precise class we can consider, it may be more useful for practical purposes to work with the class

$$
M\left(P^{*}\right)=\left\{Q: \mathcal{A}^{\prime} \rightarrow[0,1] \text { probability s.t. } Q(A) \leq P^{*}(A) \forall A \in \mathcal{A}^{\prime}\right\}
$$

of probability distributions dominated by the upper probability $P^{*}$ : this class is convex and is uniquely determined by $P^{*}$, and in some cases, it is even determined by the values of $P^{*}$ on some classes of sets (see the discussion on this subject in [20]). It becomes then interesting to investigate the relationship between these two classes, so that we can decide if the use of $P^{*}$ for modelling the information about $P_{U_{0}}$ causes an important loss of precision.

The relationship between $P(\Gamma)$ and $M\left(P^{*}\right)$ has been studied by a number of authors $([1,3,11,12,20])$, under different hypotheses on the images of the 
random set and on its initial of final spaces. We are going to study here the situation for consonant random sets. First, we investigate whether the bound given by $P^{*}(A)$ is tightest we can give for the value $P_{U_{0}}(A)$ for some arbitrary set $A$ in the final $\sigma$-field. We will use the condition of condensability, whose definition can be found in [25].

Proposition 7. Let $(\Omega, \mathcal{A}, P)$ be a probability space, $\left(X, \mathcal{A}^{\prime}\right)$ a measurable space s.t. $\mathcal{A}^{\prime}$ includes the singletons and $\Gamma: \Omega \rightarrow \mathcal{P}(X)$ a random set. If $S(\Gamma) \neq \emptyset$ and $P^{*}$ is condensable, then $P^{*}(A)=\max _{Q \in P(\Gamma)} Q(A) \forall A \in \mathcal{A}^{\prime}$.

Proof. Let $A \in \mathcal{A}^{\prime}$. Then, the condensability of $P^{*}$ implies the existence of a countable set $\left\{x_{n}\right\}_{n} \subseteq A$ s.t. $P^{*}\left(\left\{x_{n}\right\}_{n}\right)=P^{*}(A)$. Take $U \in S(\Gamma)$, and let

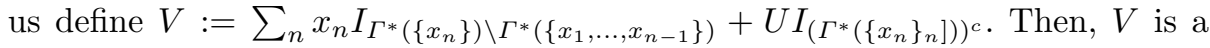
measurable selection of $\Gamma$ and moreover $P^{*}(A)=P^{*}\left(\left\{x_{n}\right\}_{n}\right)=P_{V}\left(\left\{x_{n}\right\}_{n}\right) \leq$ $\max _{Q \in P(\Gamma)} Q\left(\left\{x_{n}\right\}_{n}\right) \leq \max _{Q \in P(\Gamma)} Q(A) \leq P^{*}(A)$. Consequently $P^{*}(A)=$ $\max _{Q \in P(\Gamma)} Q(A)$.

Note that the result holds in particular for those random sets $\Gamma$ inducing a possibility measure and such that $S(\Gamma)$ is non-empty. Using this proposition, we deduce the following:

Corollary 1. Let $(\Omega, \mathcal{A}, P)$ be a probability space, $(X, d)$ be a separable metric space and let $\Gamma: \Omega \rightarrow \mathcal{P}(X)$ be a C0 random set. Then, under the weak topology, we have

1. $\operatorname{cl}\left(M\left(P^{*}\right)\right)=\operatorname{cl}(\operatorname{Conv}(P(\Gamma)))$.

2. If moreover $(\Omega, \mathcal{A}, P)$ is non-atomic, then $\operatorname{cl}(P(\Gamma))=\operatorname{cl}\left(M\left(P^{*}\right)\right)$.

Proof. From Proposition $6, \Gamma$ possesses measurable selections, and from Theorem $3, P^{*}$ is a possibility measure. Applying [19, Theorem 2.4], $P^{*}$ is condensable, and Proposition 7 implies then that $P^{*}(A)=\max _{Q \in P(\Gamma)} Q(A)$ for any $A$ in the final $\sigma$-field. The result follows now from [20, Theorems 4.4 and 4.7 ].

This shows that, in the case of $\mathrm{C} 0$ random sets, the upper probability can be used to model the information about $P_{U_{0}}$ without causing a big loss of precision. Note that the hypothesis of non-atomicity of $(\Omega, \mathcal{A}, P)$ is not too restrictive: it holds for instance in the particular case of antitone random sets, or when we know that the probability distribution of $U_{0}$ is continuous. We must warn the reader, however, that the sets $P(\Gamma)$ and $M\left(P^{*}\right)$ do not necessarily agree for $\mathrm{C} 0$ random sets, as the following example shows:

Example 2. [21, Example 3.3] Let us consider the antitone random set $\Gamma$ : $[0,1] \rightarrow \mathcal{P}([0,1])$ given by $\Gamma(\omega)=[-\omega, \omega]$. Then, the uniform probability distribution on $[-1,1]$ belongs to $M\left(P^{*}\right) \backslash P(\Gamma)$.

Therefore, the use of the upper probability in a $\mathrm{C} 0$ random set may cause some loss of information respect to the class of the probability distributions of the measurable selections. We would like to know if under some additional 
conditions we can guarantee the equality $P(\Gamma)=M\left(P^{*}\right)$. In [21], we give a number of sufficient conditions for this equality when $\Gamma$ is a random interval. Although one of those conditions (namely, that $\Gamma=[0, B]$ for some non-negative random variable $B$ ) is compatible with $\mathrm{C} 0$ random sets, the most important one (that $\Gamma=[A, B]$ with $A, B$ strictly comonotone) will only be compatible with the C0 condition when $A$ and $B$ are constant. More specifically, in the particular case where $X$ is a finite space, we have that $P(\Gamma)=M\left(P^{*}\right)$ whenever the initial probability space is non-atomic, regardless of the characteristics of the images of $\Gamma([18])$. We conclude from this that $\mathrm{C} 0$ random sets are not specially suited, when compared to other types of random sets, for modelling the imprecise observation of a random variable.

\section{Conclusions}

The approximation to consonant random sets we have considered in this paper has allowed us to prove a number of results in a fairly straightforward manner. It allows us moreover to consider consonant random sets defined between arbitrary spaces, and not necessarily antitone, because in our opinion the core of the notion of consonancy is the order we can establish in the initial space. In this respect, it would be interesting to make a deeper study of the properties of this order. We would like in particular to see if the completeness of the chain induced by a consonant random set is related to some additional condition on its images.

Concerning the different definitions of consonancy considered in this paper, we think that $\mathrm{C} 0$ random sets are sufficiently general and have moreover a number of interesting properties that other weaker notions, such as C1 and C2 random sets, do not possess in general. As an open problem from this paper, we propose to study the relationship between $\mathrm{C} 0$ and $\mathrm{C} 1$ random sets, and if a $\mathrm{C} 1$ random set inducing a possibility measure is always $\mathrm{C} 0$.

Finally, regarding the use of consonant random sets as a model for the imprecise observation of random variables, we still have to determine whether $\mathrm{C} 1$ random sets or random sets inducing a possibility measure possess measurable selections; this existence would allow us to derive a number of relationships between the class of probability distributions of these selections and those dominated by the upper probability, in the vein of Corollary 1 . We wonder if in this case the study of the chain induced on the initial space will also be helpful.

\section{References}

[1] Z. Arstein and S. Hart. Law of large numbers for random sets and allocation processes. Mathematics of Operations Research, 6(4):485-492, 1981.

[2] G. Birkhoff. Lattice theory. AMS Colloqium Publications 25, 1967.

[3] A. Castaldo, F. Macceroni and M. Marinacci. Random correspondences as bundles of random variables. Sankhya 66(3):409-427, 2004.

[4] G. de Cooman and D. Aeyels. A random set description of a possibility measure and its natural extension. IEEE Transactions on Systems, Man and Cybernetics, 30(2):124-130, 2000. 
[5] A. P. Dempster. Upper and lower probabilities induced by a multivalued mapping. Annals of Mathematical Statistics, 38:325-339, 1967.

[6] D. Dubois and H. Prade. The mean value of a fuzzy number. Fuzzy Sets and Systems, 24(3):279-300, 1987.

[7] D. Dubois and H. Prade. Possibility theory. Plenum Press, New York, 1988.

[8] D. Dubois and H. Prade. When upper probabilities are possibility measures. Fuzzy Sets and Systems, 49(1):65-74, 1992.

[9] G. Gierz, K. Hofmann, K. Keimel, J. Lawson, M. Mislove and D. Scott. A compendium of continuous lattices. Springer, Berlin, 1980.

[10] I. R. Goodman. Fuzzy sets as equivalence classes of possibility random sets. In Fuzzy Sets and Possibility Theory: Recent Developments (R. R. Yager, ed.), 327-343. Pergamon, Oxford, 1982.

[11] S. Hart and E. Köhlberg. Equally distributed correspondences. Journal of Mathematical Economics, 1(2):167-174, 1974.

[12] C. Hess. The distribution of unbounded random sets and the multivalued strong law of large numbers in nonreflexive Banach spaces. Journal of Convex Analysis, 6(1):163-182, 1999.

[13] W. Hildenbrand. Core and Equilibria of a Large Economy. Princeton University Press, Princeton, 1974.

[14] C. J. Himmelberg. Measurable relations. Fundamenta Mathematicae, 87:53-72, 1975.

[15] R. Kruse and K. D. Meyer. Statistics with vague data. D. Reidel Publishing Company, Dordretch, 1987.

[16] G. Mathéron. Random sets and integral geometry. Wiley, New York, 1975.

[17] E. Miranda, I. Couso and P. Gil. Relationships between possibility measures and nested random sets. International Journal of Uncertainty, Fuzziness and KnowledgeBased Systems, 10(1):1-15, 2002.

[18] E. Miranda, I. Couso and P. Gil. Upper probabilities and selectors of random sets. In Soft Methods in Probability, Statistics and Data Analysis (P. Grzegorzewski, O. Hryniewicz and M. A. Gil, eds.), 126-133, Physica-Verlag, 2002.

[19] E. Miranda, I. Couso and P. Gil. A random set characterisation of possibility measures. Information Sciences, 168(1-4):51-75, 2004.

[20] E. Miranda, I. Couso and P. Gil. Random sets as imprecise random variables. Journal of Mathematical Analysis and Applications, 2005, in press.

[21] E. Miranda, I. Couso and P. Gil. Random intervals as a model for imprecise information. Fuzzy Sets and Systems, 2005, in press.

[22] H. T. Nguyen. On random sets and belief functions. Journal of Mathematical Analysis and Applications, 65(3):531-542, 1978.

[23] G. L. S. Shackle. Decision, Order and Time in Human Affairs. Cambridge University Press, Cambridge, 1961.

[24] G. Shafer. A mathematical theory of evidence. Princeton University Press, New Jersey, 1976.

[25] G. Shafer. Allocations of probability. Annals of Probability, 7(5):827-839, 1979.

[26] N. Shilkret. Maxitive measures and integration. Indagationes Mathematicae, 33:109-116, 1971.

[27] M. Sugeno. Theory of fuzzy integrals and its applications, PhD Thesis, Tokyo Institute of Technology, 1974.

[28] L. A. Zadeh. Fuzzy sets as a basis for a theory of possibility. Fuzzy Sets and Systems, 1(1):3-28, 1978. 